Man and Nature

L'homme et la nature

\title{
Pour un théâtre de la présence : Diderot du théâtre au roman
}

\section{Anne Scott}

Volume 2, 1984

URI : https://id.erudit.org/iderudit/1011809ar

DOI : https://doi.org/10.7202/1011809ar

Aller au sommaire du numéro

Éditeur(s)

Canadian Society for Eighteenth-Century Studies / Société canadienne d'étude du dix-huitième siècle

ISSN

0824-3298 (imprimé)

1927-8810 (numérique)

Découvrir la revue

Citer cet article

Scott, A. (1984). Pour un théâtre de la présence : Diderot du théâtre au roman. Man and Nature / L'homme et la nature, 2, 27-36.

https://doi.org/10.7202/1011809ar
Résumé de l'article

Given the intensely dramatic reality of works like Le Neveu de Rameau and Le Paradoxe sur le comédien, why should Diderot's plays be so wooden and static? The most important reason is that Diderot's drama is constituted primarily by interaction between writer and reader, and disappears with the eighteenth-century theatre's requirement of "objective" action.
Copyright (C Canadian Society for Eighteenth-Century Studies / Sociéte canadienne d'étude du dix-huitième siècle, 1984
Ce document est protégé par la loi sur le droit d'auteur. L'utilisation des services d'Érudit (y compris la reproduction) est assujettie à sa politique d'utilisation que vous pouvez consulter en ligne.

https://apropos.erudit.org/fr/usagers/politique-dutilisation/ 
POUR UN THEATRE DE LA PRESENCE:

DIDEROT DU THEATRE AU ROMAN

Anne Scott

Que ne lui vis-je pas faire? Il pleuroit, il rioit il soupiroit; il regardoit, ou attendri, ou tranquille, ou furieux; c'etoit une femme qui se pame de douleur; c'etoit un malheureux livré a tout son desespoir; un temple qui s'eleve; des oiseaux qui se taisent au soleil couchant; des eaux qui murment (sic) dans un lieu solitaire et frais, ou qui descendent en torrents du haut des montagnes; un orage, une tempete, la plainte de ceux qui vont perir, melée au sifflement des vents, au fracas du tonnerre; c'etoit la nuit, avec ses ténèbres; c'etoit l'ombre et le silence; car le silence meme se peint par des sons. Sa tete etoit tout a fait perdue. Epuisé de fatigue, tel qu'un homme qui sort d'un profond sommeil ou d'une longue distraction; il reste immobile, stupide, etonné. Il tournoit ses regards autour de lui, comme un homme egaré qui cherche a reconnoitre le lieu où il se trouve.l

\section{Scène II}

Germeuil, Cécile, Sophie, Mademoiselle Clairet. (Sophie entre sur la scène comme une troublée. Elle ne voit point. Elle n'entend point. Elle ne sait où elle est. Cécile de son côté est dans une agitation extrême.) 
Sophie-- Je ne sais où je suis... Je ne sais où je vais... Il me semble que je marche dans les ténèbres... Ne rencontrerai-je personne qui me conduise?... O Ciel, ne m'abandonnez pas!

Germeuil (l'appelle)-- Mademoiselle, Mademoiselle.

Sophie-- Qui est-ce qui m'appelle?

Germeuil-- C'est moi, Mademoiselle, c'est moi.

Sophie-- Qui êtes-vous? Où êtes-vous? Qui que vous soyez, secourez-moi... sauvez-moi...

Germeuil (va la prendre par la main, et lui dit)-- Venez... mon enfant... Par ici.

Sophie (fait quelques pas, et tombe sur ses genoux)-- Je ne puis... La force m'abandonne... Je succombe...

Cécile-- 0 Ciel! (à Germeuil) Appelez... Eh non, n'appelez pas!

Sophie (les yeux fermés et comme dans le délire de la défaillance) -Les cruels!... Que leur ai-je fait? ${ }^{2}$

Entre ces deux extraits la différence d'un temps, le passage du passé au présent, du récit à la présentation. Deux êtres: l'un, acteur, se pose délibérément comme tel, mais se laisse persuader et emporter par son rôle, en proie à cette aliénation qui fait perdre soi à soi, ennemi mortel du comédien; l'autre ne peut que jouer une passion vraie, absente à elle-même, elle aussi aliénée. Mais les discours tenus par chacun de ces deux passages se complètent et se soutiennent: discours de la représentation, récits du corps que la raison a quitté, l'un avec sa pantomime, l'autre avec son discours et ses didascalies. Deux univers littéraires se rencontrent ici, dans l'abolition de la distinction des genres, dans un discours commun qu'anime une même esthétique de l'effet et de la persuasion, dans une même théâtralité, celle de la "parole en scène".

Partout, le théâtral fascine Diderot; l'attire, tout ce qui se montre, se joue et se révèle dans une mise en scène, structuration de l'espace, jusque dans l'orchestration de la conversation et la disposition harmonieuse des groupes dans un salon.

Or, cet être du théâtre se rêve dramaturge.

La ou plutôt les questions que nous tenterons de poser, sinon de résoudre ici, sont les suivantes:

--pourquoi, alors que toute écriture, chez Diderot, nous apparait comme dramatisation, spatialisation de la pensée discursive, opposant le simultané au consécutif, 
--alors que nous, lecteurs, sentons le besoin d'"agir", de mettre en espace, donc "en scène" Jacques le fataliste, les lettres à Sophie Volland, Le Paradoxe sur le comédien, Ceci n'est pas un conte, sans parler, bien sûr, de l'oeuvre privilégiée mais non, peutêtre, la plus propre à la mise en scène: Le Neveu de Rameau, --pourquoi Diderot, qui s'est fantasmé si sincèrement et avec une telle constance comme dramaturge, nous livre-t-il un théâtre plus figé qu'une toile de Greuze, malgré son discours d'emphase et d'emportement,

--pourquoi, enfin, Diderot semble-t-il avoir dépensé sa théâtralité ailleurs qu'entre les trois murs d'une scène?

Ces questions concernent sans doute aujourd'hui notre perception de Diderot, sur laquelle il conviendra toujours de nous arrêter, afin de ne pas perdre de vue les conditions de production de notre critique et les pressions qui déterminent les choix opérés par notre regard sur une oeuvre quelle qu'elle soit. Il convient donc, par préliminaire, de s'interroger sur les modalités de ce Diderot que l'on retrouve aux détours des analyses de Baudrillard, Serres, Kristeva ou Lyotard, stratégies d'écriture d'un philosophe des Lumières à la recherche de la vérité, mais d'abord et surtout stratégie de notre lecture. En particulier, on aime moins entendre parler aujourd'hui le Diderot dramaturge et théologien de la vertu, le Diderot bourgeois engagé dans cette part lourde, contraignante de l'idéologie des Lumières.

Ces voix qui nous gênent et font "bruit" dans ce grand corps de l'oeuvre, telle que la récupère notre propre idéologie, étaient-elles étrangères à l'oreille intérieure de celui qui se projetait dans une toile de Greuze, comme dans la scène des adieux pathétiques à sa famille avant le départ pour la Russie, composée pour les oreilles complaisantes de Devaines, de celui qui se cherchait un rôle dans le philosophe conseiller des souverains, Sénèque ambigu aux pieds de Catherine II? Essayons donc d'entendre la voix de Diderot dramaturge, autrement cette fois-ci qu'en termes d'idéologie, c'est-à-dire de tout ce qui n'est pas la nôtre, puisque ce qui est nôtre ne peut apparamment pas relever de l'idéologie...

Comment celui qui incarne ses idées dans des personnages, qui joutent par la parole, celui qui veut nous faire voir et toucher ses pensées, comment donc celui-là vit-il son expérience avec le théâtre, ce théâtre qui se joue sur scène de façon si malaisée? Les reconstructions logiques de cet échec abondent, nous ne viendrons ici qu'y ajouter les nôtres.

Esthétique du naturel, unité interne de l'action, concentration des effets, tels sont les principes que Diderot énonce pour le théâtre comme pour la peinture. Cette dernière est un rituel de répétition, une commémoration idéalisée du passé. Ainsi se joue Le fils naturel dans le salon de Dorval et Clairville. Tout, du décor aux costumes, du texte haché et d'émotion jusqu'aux gestes du sublime doit emporter, captiver le spectateur qui pense surprendre la scène sans être vu. Diderot est pleinement conscient du fait que l'action doit recevoir son "momentum" de l'intérieur et que les acteurs doivent jouer les uns pour les autres, dans l'espace circonscrit du proscenium. 
Quel étrange et unique système dramatique, en effet, que ces deux pièces qui s'insèrent dans d'autres textes, eux-mêmes dialogués et "mis en scène", qui les critiquent et les examinent à distance. La théorie se fait réflexion, défense du produit fini, nous en présente d'autres réalisations possibles, participe à une expérience totale où deux textes "se travaillent", dans l'entrelacement de la recherche et de son commentaire. Oscillation constante entre Les Entretiens et Le Fils naturel: Les Entretiens défendent-ils une pièce à laquelle l'auteur se prenait lui-même, ou bien, Le Fils naturel est-il un exercice modèle d'une nouvelle rhétorique à construire par la pratique, avec ses erreurs exemplaires qui font leçon?

Les larmes que Diderot entend faire couler pendant le spectacle de la vertu foulée aux pieds mais triomphante, seront le garanti de l'efficacité des discours; elles cimentent le pacte entre destinateur et destinataire.

L'"espèce de roman" constitué par le groupe Fils naturel, Entretiens ("histoire véritable de la pièce" dit aussi Diderot) lui permet de dépasser la scène, d'en montrer les circonstances. En même temps, cette action étendue lui permet de justifier, d'expliquer certains moments, se défendant tantôt au nom des règles dramatiques, tantôt au nom de la vérité historique. Cette question est à rattacher au problème de l'insertion des discours, de la "traduction" des disparates, aplanissement des textes que Diderot refuse, par exemple dans les discours d'André et de Rosalie, comme il le fera, dans le roman et le conte, pour celui de Suzanne dans La Religieuse ou du vieillard dans Le Supplément.

Diderot qui, hors du théâtre, refuse de pactiser avec les mots semble s'en remettre ici au vocabulaire des absolus, à l'hyperbole; le dialogue y cesse d'être la recherche même, pour représenter au lieu de se vivre et ainsi se fige dans sa fonction de véhicule d'une idéologie sans ombres.

\section{Le Théâtre est messe populaire.}

Car le thêâtre, passe-temps sublime et enfantin, accomplit sa plus belle tâche quand il sacre "peuple" la masse.

\section{Thomas Mann 3}

Le modèle de Diderot sera antique, toujours, mais la scène en sera bourgeoise ou petitement noble, et contemporaine; le sujet: la vertu cherchant partout à se faire reconnaitre.

Le travail qui sous-tend I'aventure théâtrale est celui de L'Encyclopédie; la nouvelle théologie qui doit éclairer les nations, celle de la connaissance, des lumières de l'esprit humain, ne se sépare plus d'une propédeutique de la vertu, modèle pour la société civile, que l'on installe dans des célébrations grandiosement conçues ou de simples commémorations familiales accomplies dans la ferveur et l'affection vraie. Le théâtre fera aussi pour Diderot office de machine de guerre dans la construction de l'édifice savoir/pouvoir. Depuis les voluptés partagées des rites théâtraux de Lampédouse jusqu'au spectacle surpris du salon de Dorval et Clairville, la même intention domine: élever le coeur des hommes par la contemplation de la vertu. Mieux que la rhétorique de la chaire, le langage théâtral pré- sentera une hiérophanie de la vertu, l'exaltation du sublime de l'ordinaire: 
Si j'avais l'âme, l'organe et la figure de Quinault-Dufresne, demain je monterais sur la scène, et je me tiendrais plus honoré de faire verser des larmes au méchant même, sur la vertu persécutée, que de débiter dans une chaire, en soutane et en bonnet carré, des fadaises religieuses qui ne sont intéressantes que pour les oisons qui les croient. 4

Par ce nouvel évangile, prêché selon de nouveaux accents tout de "vigueur" et de "bouillonnement", Diderot unit le sentiment de la responsabilité et la recherche d'un rapport aux autres par l'art.

Diderot rêvera d'aller toujours plus avant, toujours plus près de cette nouvelle tragédie qu'il imaginera dans le plan de Térentia en 1777; catharsis aux effets pareils au cataclysme qui fait hésiter l'être au bord tremblant de la perte de soi-même.

Entre l'outrance et l'intime vrai se cherche la dramaturgie de Diderot.

Lieu parfait parce qu'il ne connait ni les compromis ni les nuances, parce que les hésitations et les errances de la vie s'y trouvent résolues dans un ordre stable en son fond, le mélodrame fonde son économie sur la restauration d'un équilibre dont la légitimité va de soi et qui s'est trouvé pour le temps de la présentation menacé jusqu'au point de rupture. Diderot qui, dans le roman, s'efforce de rendre compte du caractère problématique de toute réalité, de la variété infinie de ses développements et de ses accents, se trouve pris ici dans les rets d'une morale moniste et dans les carcans d'un monde figé. Point de vacillement des valeurs constituées, point de tremblement au seuil du sens dans ce monde voué au salut.

Ainsi, ce qui rend Le Fils naturel non pas injouable mais difficile à recevoir comme texte convaincant, ce ne sont pas tant les éloges de la vertu et du mariage, les discours de la passion; ce n'est pas non plus seulement ce qui hésite au bord du dire: le tabou de l'inceste, mais plutôt, autour de ce même inceste si menaçant, le discours de la nature qui fait que deux êtres se reconnaissent une parenté spirituelle, si semblable à l'amour qu'elle fait chanceler l'amour même de Sophie pour Clairville qui lui préexistait, et que, tout aussi brutalement et violemment que s'en étaient fait sentir les effets, cette passion va se retourner en affection fraternelle, sans que rien ne trouble en apparence ce "cristal d'une onde pure et transparente" qu'est l'âme de Rosalie.

On pourrait également avancer que la scène première du second acte du Père de famille n'est pas "gênée" parce que le salon est encombré de personnages et que s'y déroulent deux dialogues parallèles (ce qui constitue le terrain de défense de Diderot dans le Discours sur la poésie dramatique, comme dans la lettre à Madame Riccoboni du 27 novembre 17585). Bien plutôt, notre embarras de spectateur vient de la volonté manifeste du dramaturge de nous présenter, vaille que vaille, une image monolithique de la vertu domestique. Cécile cède juste ce qu'il faut, et encore avec grâce et sagesse, aux faiblesses de son sexe et de son âge et d'Orbesson répand ses largesses avec circonspection et une dignité discrète. Le langage s'est figé dans l'emblématique de la socialité idéale. 6 
A un monde qui a perdu la notion du sacré il faut une autre sacralisation, morale et sociale cette fois, où les forces cosmiques et la dimension du destin se trouvent remplacées par l'exaltation de la vertu. Il faut construire cet univers et redonner à la parole, acte social s'il en est, valeur "théologique"; pour ce faire Diderot a recours au geste, dans la communion de la lettre et du corps.

\section{Le corps comme discours.}

Darius engagé dans la Scithie avec son armée reçoit de la part du roi des Scithes une grenouille, un oiseau, une souris et cinq flèches: le Héraut remet son présent en silence et part. Cette terrible harangue fut entendue, et Darius n'eut plus grand hâte que de regagner son pays comme il pût. Substituez une lettre à ces signes, plus elle sera menaçante moins elle effrayera; ce ne sera plus qu'une gasconade dont Darius n'aurait fait que rire.

Rousseau 7

Diderot en marche contre, contre la scène envahie de spectateurs, contre le dialogue codé et la déclamation de la scène, contre l'univers "embéguiné", "calamistré" 8 dans lequel les techniciens/technocrates du théâtre tentent de l'enfermer, cherche à retrouver le contact physique, à remplacer le langage dont la faillite est consommée. Depuis que, dans le cadre de sa réflexion sur tout ce qui est hors du champ habituel, exclu, différant, pour comprendre le "normal", Diderot s'est interrogé sur les sourds et muets, il conçoit une organisation paratactique des unités de langage pour réaliser la totalité d'effet de l'instant. Le sourd-muet produit son texte par la juxtaposition d'éléments uniques en même temps qu'univoques, dans une simultanéité, une coalescence qui conduit droit au sens. Point d'ordre contraignant là où le sens se fait d'une présence additionnée. Par le geste Diderot sent ainsi qu'il se libère de l'écriture et se rapproche de ce rêve de la présence pure, toujours plus près du centre.

Le "tableau vivant", au théâtre comme dans le roman, fera "voir" l'oreille, comme l'oeil "entendra" l'histoire que lui conte le tableau dans les Salons. Les esquisses des pantomimes, les "réclames" insérées dans La Religieuse ou Le Neveu de Rameau, comme dans le théâtre didascalies et descriptions, montrent clairement cette tendance, constante chez Diderot, à signifier l'âme par le corps, à représenter les apparences dans des tableaux, "instants parfaits" qui impliquent le spectateur dans l'illusion de l'immédiateté. Au lieu de lui fournir des bornes, le cadre semble ainsi retenu par le réseau de forces installées sur la scène. Ainsi Le père de famille s'ouvre par un tableau qui tient lieu de scène d'exposition et s'achève par une grande scène figée combinant picturalité et organisation musicale ("partition musicale" nous dit J. Chouillet, et nous pensons à la scène finale des Noces). Toute l'énergie est concentrée dans la disposition des personnages, comme dans l'aquarelle de Ziesenis conservée à la Bibliothèque Nationale, et qui représente en pyramide les groupes contrastés, arrêtés dans leur mouvement. On y voit sur un fond de gardes: à gauche, St Albin retenu ici non par Germeuil, semble-t-il, mais par un garde, et plus en avant, le Commandeur invitant l'exempt à faire son 
devoir, aux côtés de Mme Hébert et de M. Le Bon, et, à droite, Sophie aux pieds du père de famille, devant Cécile, les bras étendus, en proie à une violente émotion et Mlle Clairet un peu en retrait.

Comme la navette du conteur, le burin du graveur et le pinceau du peintre, les didascalies du dramaturge assignent sa place à chacun et font de la gestualité une dépense d'énergie, une production de sens. Ainsi selon J. Kristeva:

La gestualité, plus que le discours (phonétique) ou l'image (visuelle) est susceptible d'être étudiée comme une activité dans le sens d'une dépense, d'une productivité antérieure (...) à la représentation comme phénomène de signification...9

$\mathrm{Au}$ lever du rideau le spectateur se change en réceptivité pure, protégé par l'ombre et le silence; son espace aussi inviolable que la scène, il attend qu'on lui montre une action. Ainsi Diderot conçoit un théâtre où les acteurs donnent à tout instant l'impression de jouer pour eux seuls dans le cercle de leur propre action, alors que, dans le roman, le narrateur met incessamment le lecteur en question. Le narrateur s'y suscite un lecteur, lui souffle ses réponses, bref en fait sa chose qu'il intègre à sa création et entend manipuler à son gré. Le public de théâtre est, lui, cet ensemble changeant, incertain, qui peut un jour faire tomber ce qu'il acclamait la veille, une foule dont le goût est à éduquer. Là réside un des grands problèmes de la dramaturgie de Diderot, puisque c'est dans le rapport au spectateur, pourtant passager clandestin à la fête du sens, que le philosophe fait entendre sa voix propre, s'installe, lui, sur la scène. Diderot, qui est si bien convaincu que l'essentiel de l'illusion dramatique est le sentiment de se trouver là par hasard, dans une action domestique, spectacle en apparence gratuit, ce même Diderot ne peut renoncer à parler sa propre vérité.

Dans la mesure où le discours théâtral est discours d'un sujet scripteur, il est discours d'un sujet immédiatement dessaisi de son Je... le discours théâtral est un discours sans sujet.10

Ce sujet énonçant, Le Paradoxe ou la recherche philosophique partout le met en scène. C'est pour cela que la parole-action du dialogue romanesque, qui questionne l'illusoire de l'univers créé et l'entreprise même de l'écriture, convient si bien à Diderot qui veut agir, engager son interlocuteur dans des procédures de séduction et de légitimation de son discours. Dans le roman, il peut entrer et sortir à son gré, sans être jamais figé dans un rôle ou une absence. Il n'en est rien au théâtre. En vain il s'efforce de "traduire" pour nous les actions des personnages, dont le caractère véridique est ensuite discuté dans Les Entretiens et le Discours sur la poésie dramatique, comme le seront certaines remarques de Jacques ou les adieux du vieillard dans Le Supplément.

Dans Est-il bon? est-il méchant? c'est finalement le sujet énonçant qu'il met en scène. Est représenté dans cette pièce le travail de soi pour ressaisir son unité en même temps que l'acceptation bougeonne de la perte de soi au profit des autres. La pièce présente un univers atomisé, un monde au centre absent, une problématique non surmontée, dans lequel l'ironie 
redonne à tout et à tous sa juste place et où au bout du compte le logos se fait propédeutique et la réflexion heuristique dans le persiflage même, persiflage qui n'est pas sans nous rappeler le Neveu quand il se fait roublard et flagorneur.

Revenons maintenant à la question initiale qui nous préoccupait d'entrée de jeu: comment Diderot qui a si bien compris les règles de fonctionnement de la machine théâtrale, nous présente-t-il un théâtre figé, des pôles irréductibles? Où situer alors la théâtralité de Diderot? Serat-elle à chercher sur la scène projetée, installée, campée par le romancier, ou sur celle fantasmée par le dramaturge, "aurige" de ses contemporains en mal de vertu? A l'intérieur du cercle magique? du "cadre" du café de la Régence? du couvent d'Arpajon, du théâtre de Saint-Germain ou de la Comédie Française? Ou, plus largement et plus précisément, sur la scène du texte, celle qui s'installe parce que se rencontrent deux instances de parole, parce que s'y vit la dépense d'une énergie langagière, que s'y affrontent des êtres de parole dont le combat tisse l'espace?

A propos du Paradoxe sur le comédien, Philippe Lacoue-Labarthe écrit:

L'auteur--Diderot--occupe donc simultanément (je veux dire: dans le même texte) deux places. Et deux places incompatibles. Il est le Premier, l'un des deux interlocuteurs. Ou tout au moins il s'est donné pour tel. Mais il est également celui qui, se mettant ouvertement en position d'auteur ou d'énonciateur général, se démarque du Premier ou peut, ne serait-ce que par jeu, s'en démarquer et le constituer en personnage. 11

et il ajoute:

L'intrusion du je..., loin de signifier l'appropriation ou la réappropriation maitrisante du texte par l'auteur, ... représente le moment où le texte (le livre entier) vacille dans son statut. 12

Pourquoi ses deux places seraient-elles incompatibles, puisque la seule place assignable du je est le texte qui s'écrit, place non représentée dans son discours, mais constitué par le travail même de l'écriture. Ceci est vrai, à notre point de vue, de l'ensemble de l'oeuvre qui se présente dans sa totalité comme le lieu inassignable du sujet énonçant. Le je-narrateur (que ce soit dans Le Paradoxe, dans Le Supplément ou dans Le Neveu) n'a pas à avouer être quiconque, pas plus le philosophe-Moi, l'interlocuteur de la raison et du juste milieu, pas plus Ariste que le fou, le dissident, le monstre moral ou le scélérat qui fascine par son ex-centricité. Le jeénonçant donne voix au dialogue de la pensée dont la vie propre, l'essence même est toujours chez Diderot de l'ordre de l'agonistique, donc de l'ordre de la représentation et de la théâtralité, et là se love le serpent au coeur de l'entreprise spécifiquement dramatique de Diderot, cet inassignable du lieu de la parole qui fait que le je-énonçant s'immisce en tout son texte.

Ce qui est unique et passionnant chez Diderot c'est bien la problématique des formes de la présence. Le scripteur ne peut s'absenter de son 
texte et toute la fascination pour nous vient de ce vacillement de la présence, de cette mouvance perpétuelle de l'espace d'où parle le je: mais cela nous met mal à l'aise aussi, obsédés que nous sommes par l'assignation d'un lieu et d'une voix.

Au congrès de Toronto consacré en 1980 au théâtre,13 on avait beaucoup parlé du "jeu", jeu des acteurs, jeux de scène, jeux de lumières et d'images, jeu des lecteurs aussi, jeu de nos jouissances, mais on avait bien peu fait allusion à cette forme particulière et essentielle du jeu, cette respiration, ce vide, cette marge et ce défaut de serrage qui crée tensions et libertés. 14 Cet espace de jeu, Diderot le cherche dans le roman dégagé de sa tâche didactique, dans cette présence qui, en face, "réclame la brutalité d'un surgissement et la frémissante disponibilité de l'autre," selon le mot de Roger Kempf. 15 L'auteur accuse son épaisseur de scripteur, ne peut s'effacer devant son texte, y entre comme force agissante de persuasion. Il intervient en personne, tel un catalyseur, dans la relation entre le lecteur et le texte, prétend distribuer toutes les cartes du jeu et donner le sens, à la fois message et pulsion orientée. Engagement total de l'être dans l'acte d'écriture, présence intransigeante du scripteur, tout entier pris dans l'instant de la création. Ce "bonheur" de Diderot, la jouissance de l'instant, explique le caractère de son écriture, ce dynamisme qui prend des airs de désordre, "cette jubilation continue, faite d'une sommation d'instants parfaits" dont parle R. Barthes. 16

Cette présence envahissante de l'auteur au coeur du texte, ce discours objectif, tenu dans le théâtre sur les personnages mêmes qui nuit à la création de l'illusion dramatique, y installe les deux pôles de la communication dont la relation de la parole. Par l'esthétique du dévoilement Diderot tente de dépasser la problématique de son difficile effacement en tant qu'instance énonciatrice. Au coeur de ce dialogue il reste cette parole aux cents visages.

Voix puissante au sein des "paroles mêlées".17

Université de la Colombie-Britannique

NOTES

1 Le Neveu de Rameau, édition critique de Jean Fabre (Genève: Droz, 1963), pp. 84-85.

2 Le Père de famille, Acte III, scène 2, dans Oeuvres complètes (Paris: Hermann, 1980), X, 250-51.

3 Thomas Mann, Essai sur le théâtre, inédit en France, publié pour la première fois sous le titre Versuch uber das Theater dans Nord und Sud, 370 et 371 (janv, et fév. 1908), Cahiers Renaud-Barrault, 98 (1979), p. 153. 
4 Lettre à Mademoiselle Jodoin, 21 février 1763, in Oeuvres complètes, édition R. Lewinter (Paris: Le Club Français du Livre, 1970), VII, 651 .

5 Edition Lewinter, III, 674.

6 Le Père de famille, Acte II, scène 1.

7 Essai sur l'origine des langues, édition Ch. Porset (Paris: Nizet, 1976), pp. 31, 33 .

8 Lettre à Madame Riccoboni, Lewinter, III, 679.

9 Julia Kristeva, Séméiotikè: Recherches pour une sémanalyse (Paris: Seuil, 1969), p. 93.

10 Anne Ubersfeld, Lire le théâtre (Paris: Les Classiques du peuple, 1978), p. 264.

11 Philippe Lacoue-Labarthe, "Diderot, le paradoxe et la mimésis, "Poétique, 4 (sept. 1980), 269.

12 Lacoue-Labarthe, 269.

13 Toronto, Département de français, 14-16 novembre 1980, Colloque sur la théâtralité.

14 "Tension du jeu avec l'histoire, tension aussi du jeu avec la présence. Le jeu est la disruption de la présence... Le jeu est toujours jeu d'absence et de présence, mais si l'on veut le penser radicalement, il faut le penser avant l'alternative de la présence et de l'absence; il faut penser l'être comme présence ou absence à partir de la possibilité du jeu et non l'inverse." J. Derrida, L'Ecriture et la différence (1967; réimp. Paris: Seuil, Collection Points, 1979), p. 426.

15 Roger Kempf, Diderot et le roman ou le Démon de la présence (Paris: Seuil, 1964), p. 47 .

16 Roland Barthes, "Diderot, Brecht, Eisenstein," Revue d'esthétique (1973), p. 188.

17 Jean Peytard, "Les Chants de Maldoror et 1 'univers mythique de Lautréamont," La Nouvelle Critique, no. 37 (octobre 1970), 51. 